NASA/CR-1998-208447

ICASE Report No. 98-27

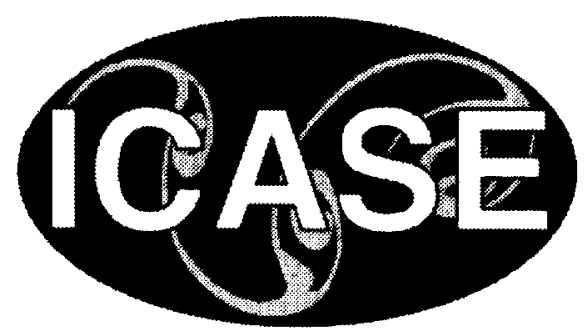

\title{
On the Circulation Manifold for Two Adjacent Lifting Sections
}

Luca Zannetti and Angelo Iollo

Dipartimento di Ingegneria Aeronautica e Spaziale, Torino, Italy

Institute for Computer Applications in Science and Engineering NASA Langley Research Center

Hampton, VA

Operated by Universities Space Research Association

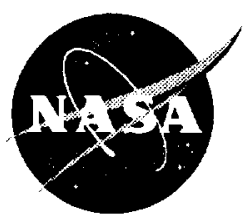

National Aeronautics and

Space Administration

Langley Research Center

Prepared for Langley Research Center under

Hampton, Virginia 23681-2199

Contracts NAS1-19480 and NAS 1-97046

July 1998 
Available from the following:

NASA Center for AeroSpace Information (CASI) 7121 Standard Drive

Hanover, MD 21076-1320

(301) 621-0390
National Technical Information Service (NTIS) 5285 Port Royal Road

Springfield, VA 22161-2171

(703) $487-4650$ 


\title{
ON THE CIRCULATION MANIFOLD FOR TWO ADJACENT LIFTING SECTIONS *
}

\author{
LUCA ZANNETTI ${ }^{\dagger}$ AND ANGELO IOLLO ${ }^{\ddagger}$
}

\begin{abstract}
The circulation functional relative to two adjacent lifting sections is studied for two cases. In the first case we consider two adjacent circles. The circulation is computed as a function of the displacement of the secondary circle along the axis joining the two centers and of the angle of attack of the secondary circle. The gradient of such functional is computed by deriving a set of elliptic functions with respect both to their argument and to their period. In the second case studied, we considered a wing-fiap configuration. The circulation is computed by some implicit mappings, whose differentials with respect to the variation of the gcometrical configuration in the physical space are found by divided differences. Configurations giving rise to local maxima and minima in the circulation manifold are presented.
\end{abstract}

Key words. potential flow, elliptic functions, optimal shape design.

Subject classification. Fluid Mechanics, Applied and Numerical Mathematics

1. Introduction. Considering the growth of research and industrial interest in aerodynamic shape optimization, we felt that a reference test case amenable to analytic solution and yet complex enough to be representative of problems arising from real world applications, was lacking. For this reason we propose in this paper two optimization test cases based on the potential flow solution. The first is represented by the problem of finding an extremum of the circulation manifold relative to the flow about two circles, when the secondary circle and its trailing edge are displaced. The global circulation and its gradient are computed by analytical tools. In the second test case, the problem of computing the circulation and its gradient for the flow about two airfoils, when the second airfoil is translated and rotated with respect to the first one, is solved.

The solution is determined by a classical analytical technique that requires two steps. The first step, which is common to both test cases, is the determination of the flow solution in the plane of the two circles. In the plane of the airfoils only 3 parameters are varied: the position of the leading edge of the flap $\left(X_{s}, Y_{s}\right)$ and its deflection $\Theta$. On the plane of the circles it is shown that there are 6 parameters changing as the flap is moving in the physical space. The six parameters are the modulus and direction of the speed at infinity, the radii of the the two circles and the angles defining the position of the two trailing edges. Using the Lagally [1] solution for the flow about two circles, it was possible to compute the analytical differential of the total circulation about the two circles. The second solution step is the transformation of the two airfoils into two circles using a Theodorsen transform for the primary airfoil and a Garrick transform for the other. Any two airfoils can be transformed by this technique. Yet, the mappings used are known implicitly by means of a FFT. Therefore, for simplicity, the Jacobian of these transformations with respect to the translation and rotation of the flap is computed by divided differences. Such differentiation can be conducted to any order of accuracy at a very limited computational cost.

*This work was partially supported by the National Aeronautics and Space Administration under NASA Contract Nos. NAS1-19480 and NAS1-97046 while the authors were in residence at the Institute for Computer Applications in Science and Engineering (ICASE), NASA Langley Research Center, Hampton, VA 23681-2199.

${ }^{\dagger}$ Dipartimento di Ingegneria Aeronautica e Spaziale, Politecnico di Torino, 10129, Torino, Italy.

$\ddagger$ This work was partially supported by the European Commission under the Marie Curie Fellowship Program. Dipartimento di Ingegneria Acronautica e Spaziale, Politecnico di Torino, 10129, Torino, Italy. Present address: INRIA - Sophia Antipolis, Projet SINUS, Sophia Antipolis Cedex, France. 
Optimization consists in defining a functional which represent the cost (or merit) of a solution, as a function of the geometrical configuration. The optimal solution is the minimum (or the maximum) of such a functional. An optimization algorithm is based on three ingredients: the flow solver, a routine to compute the gradient, and an optimizer, i.e., a strategy to march toward the extremum of the functional, see for example [2] and references therein. Using the exact computation of the gradient that we propose it is possible to validate the accuracy of numerical gradients obtained by adjoint or sensitivity analysis formulations. Furthermore, general type descent algorithms can be tested using the exact gradient given in this paper, in order to evaluate performance in the context of acrodynamic optimization. For example, the effects of local minima on the performance of various optimizers can be investigated. There was another attempt at computing an exact test case for the circulation about wing-flap section configurations [3]. It was found that the derivation presented therein is based on some erroneous assumptions. In fact, because of the motion of the flap, the primary airfoil shape and the flap shape are deformed, making that test case of no practical relevance.

Section 2 is devoted to the study of the analytical gradient of the circulation about two adjacent circles with given trailing edge. A first exact test case is presented. In Section 3 the mapping from the two airfoils plane to the two circles plane is sketched. Finally, he results :elative to the second test case proposed, obtained by divided differences, are presented.

2. The circulation manifold of two adjacent circles. In this section we will assume that the two profiles are indeed two circles for the reason that in this case it is possible to determine by analytical differentiation the dependence of the circulation and hence of the lift, as a function of some geometrical parameters.

Consider two circles (Fig. 2.1-2.3), the first is the unit circle centerd at the origin, the second has radius $r_{s}$ and center at $x=x_{s}$ on the rcal axis of the plane $z=x+i y$. The two circles are immersed in a potential incompressible flow. The speed at infinity of such flow is $q_{\infty}=\left|q_{c_{\infty}}\right| e^{i \alpha_{0}}$ and the Kutta condition is imposed at the points $z_{p}$ and $z_{s}$, which belong to the primary and secondary circle respectively. They are determined by $\varphi_{p}=\arg \left(z_{p}\right)$ and $\varphi_{s}=\arg \left(z_{s}\right)$. The solution to this flow field is due to Lagally [1] and Ferrari [4].

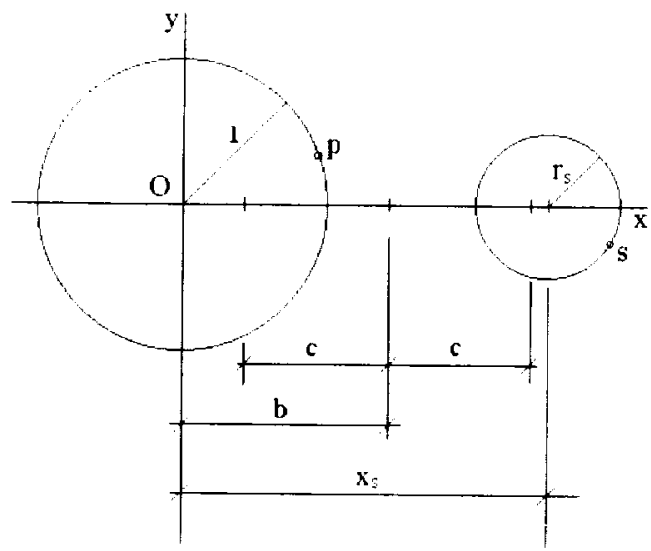

FIG. 2.1. Plane of the two circies.

Let

$$
b=\frac{x_{s}^{2}-r_{s}^{2}+1}{2 x_{s}} \quad c=\sqrt{\left(x_{s}-b\right)^{2}-r_{s}^{2}}
$$




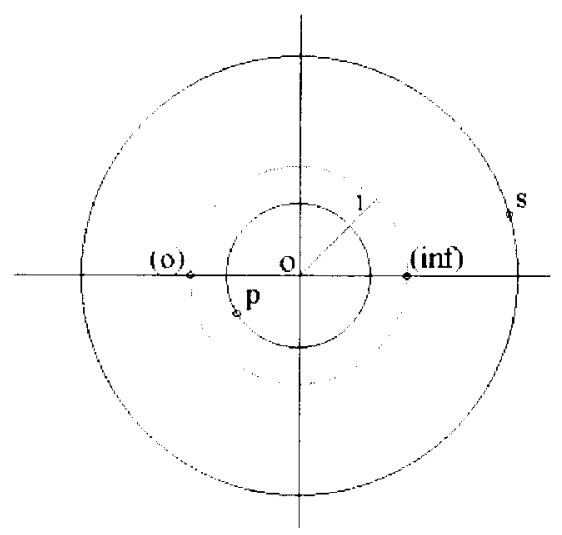

FIG. 2.2. Plane of the concentric circles.

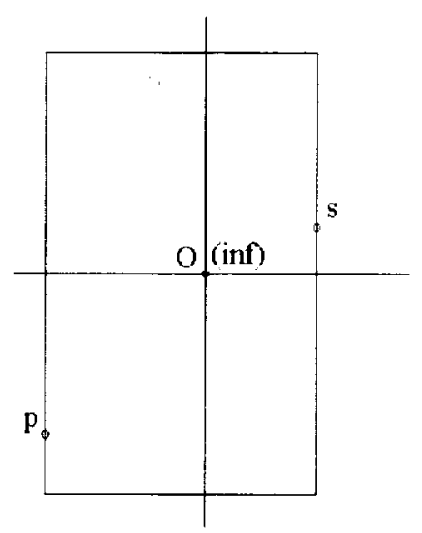

FIG. 2.3. Plane of the rectangle $B$.

The two circles considered belong to a family of Apollonius circles with focal points at $c+b$ and $c-b$ on the real axis. The mapping

$$
\nu=\frac{z-b}{c}
$$

transforms this Apollonius manifold to another, whose focal points are at $\nu=-1$ and $\nu=1$.

Let us exploit the invariance of the complex potential $w$ under conformal mappings, and let us consider the flow on the transformed plane $\nu$. The complex velocity at infinity is

$$
Q_{\infty}^{*}=\left|Q_{\infty}\right| e^{-i \beta_{0}}=\lim _{\nu \rightarrow \infty} \frac{d w}{d \nu}=\lim _{z \rightarrow \infty} \frac{d w}{d z} \frac{d z}{d \nu}=c\left|q_{\infty}\right| e^{-i \alpha_{0}}
$$

The bilinear transform

$$
\mu=\frac{\nu+1}{\nu-1}
$$

maps the two circles in the plane $z$ onto two concentric circles with center in the origin. The internal circle, whose radius is $r_{i}<1$, corresponds the primary circle, the external, whose radius is $r_{e}>1$, corresponds to the secondary circle. The region bounded by the two circles corresponds to the region where the flow field 
takes place on $z$ and the point $\mu=1$ corresponds to infinity on the plane $z$ as well as on $\nu$. The trailing edges $z_{p}$ and $z_{s}$ are mapped to $\mu_{p}=r_{i} \exp \left(i \vartheta_{p}\right)$ and $\mu_{s}=r_{e} \exp \left(i \vartheta_{s}\right)$ respectively.

Now, the mapping

$$
\lambda=\log \mu
$$

transforms the region bounded by the concentric circles into a periodic row of rectangles in the plane $\lambda=\xi+i \eta$. We denote by $B$ the rectangle $-\beta \leq \xi<\alpha$ and $-\pi \leq \eta<\pi$, with $\beta=-\log r_{i}$ and $\alpha=\log r_{e}$. The period of this row of rectangles is $2 i \pi$. The primary circle goes onto the segment $\xi=-\beta,-\pi \leq \eta<\pi$, while the secondary circle goes onto the segment $\xi=\alpha,-\pi \leq \eta<\pi$. The trailing edges are mapped to $\lambda_{p}=-\beta+i \vartheta_{p}$, and $\lambda_{s}=\alpha+i \vartheta_{s}$.

The complex speed inside $B$ is singular only at the origin $\lambda==0$, which corresponds to infinity in $\nu$ and $z$. The possible singularities can be a pole of order 2, responsible for the far field speed on the physical plane, and a pole of order 1, relative to a possible vortex at infinity whose intensity is equal to the opposite of the circulation about the two circles. In addition, the complex speed, which is periodic in the direction of the imaginary axis with period $2 i \pi$, must be periodic in the direction of the real axis with period $2(\alpha+\beta)$ in order to satisfy the impermeability condition at the walls of the two circles. As a matter of fact, the impermeability condition is obtained by successive reflections with respect to the two sides of the rectangle, so that it is periodic with period $2(\alpha+\beta)$ in that direction. Therefore, the period is formed by $B$ and the mirror image of $B$. We conclude that the complex speed is expressed by an elliptic function with scrni-periods $\omega=\alpha+\beta$ and $\bar{\omega}=i \pi$, and that it has poles of order 1 and 2 located in $\lambda=0$ and $\lambda=-2 \beta$. Hence, the complex speed is

$$
\frac{d w}{d \lambda}=-\frac{i \Gamma}{2 \pi}[\zeta(\lambda)-\zeta(\lambda+2 \beta)]-2\left[Q_{\infty}^{*} \wp(\lambda)-Q_{\infty} \wp(\lambda+2 \beta)\right]+i \kappa
$$

and, therefore, the complex potential is

$$
w=-\frac{i \Gamma}{2 \pi} \log \frac{\sigma(\lambda)}{\sigma(\lambda+2 \beta)}+2\left[Q_{\infty}^{*} \zeta(\lambda)-Q_{\infty} \zeta(\lambda+2 \beta)\right]+i \kappa \lambda
$$

where $\zeta, \wp, \sigma$, arc Weicrstrass functions with semi-periods $\omega=\alpha+\beta, \bar{\omega}=i \pi$.

The constants $\Gamma$ and $\kappa$ are respectively the global circulation and the circulation about the secondary circle. They are determined by imposing the Kutta conditions

$$
\left(\frac{d w}{d \lambda}\right)_{\lambda=\lambda_{p}}=\left(\frac{d w}{d \lambda}\right)_{\lambda=\lambda_{s}}=0
$$

We have

$$
\Gamma=\frac{-4 \pi i\left(C_{s}-C_{p}\right)}{A_{p}-A_{s}}, \quad \kappa=\frac{\Gamma}{2 \pi} A_{p}-2 i C_{p}
$$

where

$$
\begin{aligned}
& C_{s}=Q_{\infty}^{*} \wp\left(\lambda_{s}\right)-Q_{\infty} \wp\left(\lambda_{s}-2 \beta\right) \\
& C_{p}=Q_{\infty}^{*} \wp\left(\lambda_{p}\right)-Q_{\infty} \wp\left(\lambda_{p}+2 \beta\right) \\
& A_{s}=\zeta\left(\lambda_{s}\right)-\zeta\left(\lambda_{s}+2 \beta\right) \\
& A_{p}=\zeta\left(\lambda_{p}\right)-\zeta\left(\lambda_{p}+2 \beta\right)
\end{aligned}
$$


2.1. Differentiation. Two adjacent airfoils can always be mapped using the Theodorsen-Garrick transform onto two circles belonging to an Apollonius family of circles on the complex plane $\nu$, with focal points at $\nu=-1$ and $\nu=1$. When either the geometric or the flow configuration of the two airfoils changes, 6 parameters may change on the plane of the two circles

$$
\left|Q_{\infty}\right|, \quad \beta_{0}, \quad \alpha, \quad \beta, \quad \vartheta_{s}, \quad \vartheta_{p}
$$

These parameters, in turn, determine the circulation $\Gamma$ (see Eq. (2.9)) and hence the lift. Consider the problem of two adjacent rigid airfoils, the lift is a function of 4 parameters: the angle of attack and the three degrees of freedom of the second airfoil (every geometric or flow configuration can be reduced to the case of fixed primary profile and $q_{\infty}=1$ ). Therefore kecping constant the angle of attack at $\infty$, in the easiest case of two circular airfoils the degrees of freedom to be considered are 3: the distance between the centers $x_{s}$, and the position of the two trailing edges $\varphi_{s}, \varphi_{p}$. In such simple case the circulation (and the lift) is a 3 parameter manifold in a 6-dimensional space represented by the parameters (2.11). Therefore, in general we can express all derivatives of the circulation as a combination of the derivatives of Eqs. (2.10) with respect to the parameters (2.11).

Since $\omega=\alpha+\beta$, to differentiate with respect to $\alpha$ or $\beta$ implies differentiation with respect to the semiperiod of the elliptic functions $\omega$. These derivatives are given in Section 2.2 .

\subsubsection{Derivative $\partial_{\alpha}$.}

$$
\begin{aligned}
\frac{\partial C_{s}}{\partial \alpha} & =Q_{\infty}^{*}\left[\wp^{\prime}\left(\lambda_{s}\right)+\frac{\partial \wp\left(\lambda_{s}\right)}{\partial \omega}\right]-Q_{\infty}\left[\wp^{\prime}\left(\lambda_{s}+2 \beta\right)+\frac{\partial \wp\left(\lambda_{s}+2 \beta\right)}{\partial \omega}\right] \\
\frac{\partial C_{p}}{\partial \alpha} & =Q_{\infty}^{*} \frac{\partial \wp\left(\lambda_{p}\right)}{\partial \omega}-Q_{\infty} \frac{\partial \wp\left(\lambda_{p}+2 \beta\right)}{\partial \omega} \\
\frac{\partial A_{s}}{\partial \alpha} & =\left[-\wp\left(\lambda_{s}\right)+\frac{\partial \zeta\left(\lambda_{s}\right)}{\partial \omega}\right]-\left[-\wp\left(\lambda_{s}+2 \beta\right)+\frac{\partial \zeta\left(\lambda_{s}+2 \beta\right)}{\partial \omega}\right] \\
\frac{\partial A_{p}}{\partial \alpha} & =\frac{\partial \zeta\left(\lambda_{p}\right)}{\partial \omega}-\frac{\partial \zeta\left(\lambda_{p}+2 \beta\right)}{\partial \omega}
\end{aligned}
$$

\subsubsection{Derivative $\partial_{\beta}$.}

$$
\begin{aligned}
& \frac{\partial C_{s}}{\partial \beta}=Q_{\infty}^{*} \frac{\partial \wp\left(\lambda_{s}\right)}{\partial \omega}-Q_{\infty}\left[2 \wp^{\prime}\left(\lambda_{s}+2 \beta\right)+\frac{\partial \wp\left(\lambda_{s}+2 \beta\right)}{\partial \omega}\right] \\
& \frac{\partial C_{p}}{\partial \beta}=Q_{\infty}^{*}\left[-\wp^{\prime}\left(\lambda_{p}\right)+\frac{\partial \wp\left(\lambda_{p}\right)}{\partial \omega}\right]-Q_{\infty}\left[\wp^{\prime}\left(\lambda_{p}+2 \beta\right)+\frac{\partial \wp\left(\lambda_{p}+2 \beta\right)}{\partial \omega}\right] \\
& \frac{\partial A_{s}}{\partial \beta}=\frac{\partial \zeta\left(\lambda_{s}\right)}{\partial \omega}-\left[-2 \wp\left(\lambda_{s}+2 \beta\right)+\frac{\partial \zeta\left(\lambda_{s}+2 \beta\right)}{\partial \omega}\right] \\
& \frac{\partial A_{p}}{\partial \beta}=\wp\left(\lambda_{p}\right)+\frac{\partial \zeta\left(\lambda_{p}\right)}{\partial \omega}-\left[-\wp\left(\lambda_{p}+2 \beta\right)+\frac{\partial \zeta\left(\lambda_{p}+2 \beta\right)}{\partial \omega}\right]
\end{aligned}
$$

2.1.3. Derivative $\partial_{\vartheta_{s}}$.

$$
\begin{aligned}
& \frac{\partial C_{s}}{\partial \vartheta_{s}}=i\left[Q_{\infty}^{*} \wp^{\prime}\left(\lambda_{s}\right)-Q_{\infty} \wp^{\prime}\left(\lambda_{s}+2 \beta\right)\right] \\
& \frac{\partial C_{p}}{\partial \vartheta_{s}}=0 \\
& \frac{\partial A_{s}}{\partial \vartheta_{s}}=i\left[-\wp\left(\lambda_{s}\right)+\wp\left(\lambda_{s}+2 \beta\right)\right] \\
& \frac{\partial A_{p}}{\partial \vartheta_{s}}=0
\end{aligned}
$$


2.1.4. Derivative $\partial_{\vartheta_{p}}$.

$$
\begin{aligned}
& \frac{\partial C_{s}}{\partial \vartheta_{P}}=0 \\
& \frac{\partial C_{p}}{\partial \vartheta_{s}}=i\left[Q_{\infty}^{*} \wp^{\prime}\left(\lambda_{P}\right)-Q_{\infty} \wp^{\prime}\left(\lambda_{P}+2 \beta\right)\right] \\
& \frac{\partial A_{s}}{\partial \vartheta_{s}}=0 \\
& \frac{\partial A_{p}}{\partial \vartheta_{s}}=i\left[-\wp\left(\lambda_{P}\right)+\wp\left(\lambda_{P}+2 \beta\right)\right]
\end{aligned}
$$

2.1.5. Derivative $\partial_{\left|Q_{\infty}\right|}$.

$$
\begin{aligned}
\frac{\partial C_{s}}{\partial\left|Q_{\infty}\right|} & =\frac{C_{s}}{\left|Q_{\infty}\right|} \\
\frac{\partial C_{p}}{\partial\left|Q_{\infty}\right|} & =\frac{C_{p}}{\left|Q_{\infty}\right|} \\
\frac{\partial A_{s}}{\partial\left|Q_{\infty}\right|} & =0 \\
\frac{\partial A_{p}}{\partial\left|Q_{\infty}\right|} & =0
\end{aligned}
$$

2.1.6. Derivative $\partial_{\beta_{0}}$.

$$
\begin{aligned}
& \frac{\partial C_{s}}{\partial \beta_{0}}=-i\left[Q_{\infty}^{*} \wp\left(\lambda_{s}\right)+Q_{\infty} \wp\left(\lambda_{s}+2 \beta\right)\right] \\
& \frac{\partial C_{p}}{\partial \beta_{0}}=-i\left[Q_{\infty}^{*} \wp\left(\lambda_{p}\right)+Q_{\infty \wp}\left(\lambda_{p}+2 \beta\right)\right] \\
& \frac{\partial A_{s}}{\partial \beta_{0}}=0 \\
& \frac{\partial A_{p}}{\partial \beta_{0}}=0
\end{aligned}
$$

2.2. Derivative of the Weierstrass functions with respect to the period. When the geometric configuration varies on the physical plane, the radii $r_{i}, r_{e}$ change as well. Therefore on the plane $\lambda$ the semiperiod $\omega=\alpha+\beta$ of the elliptic functions $\wp(u ; \omega, \bar{\omega})$ and $\zeta(u ; \omega, \bar{\omega})$ of Weierstrass varies when the geometric configuration is changing. It follows that in the derivatives of Eqs. (2.12-2.13) with respect to $\alpha$ and $\beta$, besides the partial derivatives of $\wp(u ; \omega, \bar{\omega})$ and $\zeta(u ; \omega, \bar{\omega})$ with respect to $u$, there appear the derivatives with respect to $\omega$. For example, consider the derivative with respect to $\alpha$ of $\wp\left(\lambda_{s}\right)$, appearing in $\partial_{\alpha} C_{s}$. Since $\lambda_{s}=\alpha+i \vartheta_{s}$, we have

$$
\frac{\partial \wp\left(u=\alpha+i \vartheta_{s} ; \omega=\alpha+\beta, \bar{\omega}=i \pi\right)}{\partial \alpha}=\frac{\partial \wp(u ; \omega, \bar{\omega})}{\partial u} \frac{\partial u}{\partial \alpha}+\frac{\partial \wp(u ; \omega, \bar{\omega})}{\partial \omega} \frac{\partial \omega}{\partial \alpha}
$$

We mention in passing that

$$
\frac{\partial \zeta(u)}{\partial u}=-\wp(u), \quad \frac{\partial \wp(u)}{\partial u}=\wp^{\prime}(u)
$$

On the contrary, the derivation of these functions with respect to the semi-period is not as easy.

It is convenient both for the computation and for the derivition to express $\zeta(u)$ and $\wp(u)$ in terms of Jacobi $\vartheta_{\alpha}(v)$ functions [5]:

$$
\begin{aligned}
& \zeta(u)=\frac{1}{2 \omega} \frac{\vartheta_{1}^{\prime}(v)}{\vartheta_{1}(v)}+2 \eta v \\
& \wp(u)=e_{1}+\frac{1}{4 \omega^{2}}\left[\frac{\vartheta_{1}^{\prime}(0)}{\vartheta_{2}(0)} \frac{\vartheta_{2}(v)}{\vartheta_{1}(v)}\right]^{2}
\end{aligned}
$$


with

$$
\begin{aligned}
v & =\frac{u}{2 \omega} \\
\eta & =-\frac{1}{12 \omega} \frac{\vartheta_{1}^{\prime \prime \prime}(0)}{\vartheta_{1}^{\prime}(0)} \\
e_{1} & =\frac{1}{12}\left(\frac{\pi}{\omega}\right)^{2}\left[\vartheta_{3}^{4}(0)+\vartheta_{4}^{4}(0)\right]
\end{aligned}
$$

wherc' denotes derivation with respect to $v$. Jacobi $\vartheta_{\alpha}(v)$ functions can be expressed as

$$
\begin{aligned}
& \vartheta_{1}(v)=2 q^{\frac{1}{4}} \sum_{n=0}^{\infty}(-1)^{n} q^{n(n+1)} \sin [(2 n+1) \pi v] \\
& \vartheta_{2}(v)=2 q^{\frac{1}{4}} \sum_{n=0}^{\infty} q^{n(n+1)} \cos [(2 n+1) \pi v] \\
& \vartheta_{3}(v)=1+2 \sum_{n=1}^{\infty} q^{n^{2}} \cos (2 n \pi v) \\
& \vartheta_{4}(v)=1+2 \sum_{n=1}^{\infty}(-1)^{n} q^{n^{2}} \cos (2 n \pi v)
\end{aligned}
$$

with

$$
q=e^{i \pi \tau}, \quad \tau=\frac{\bar{\omega}}{\omega}
$$

The convergence rate of the series in Eqs. (2.24) is high when $|\omega| \leq|\omega|$ and $\operatorname{Im}(\tau)>0$. It is always possible to satisfy such conditions with a proper choice of the rectangle periods. Up to now we always assumed $\omega=\alpha+\beta, \bar{\omega}=i \pi$, and hence the first condition is not always met. If it turns out that $|\omega|>|\bar{\omega}|$, it is possible to use the allowed transformations for the periods of clliptic functions (see [5]), as the periods are not uniquely defined. In our case we can define new semi-periods $\dot{\omega}$ and $\dot{\bar{\omega}}$ which are obtained from the previous by means of a transformation of first order

$$
\dot{\omega}=\bar{\omega}, \quad \dot{\bar{\omega}}=-\omega
$$

so that the conditions mentioned are both satisfied.

Finally, we obtain

$$
\begin{gathered}
\frac{\partial \zeta(u)}{\partial \omega}=\frac{1}{2 \Omega \vartheta_{1}(v)}\left[-J \frac{u}{2 \Omega^{2}} \vartheta_{1}^{\prime \prime}(v)+\frac{\partial \vartheta_{1}^{\prime}(v)}{\partial \omega}\right]- \\
+\frac{\vartheta_{1}^{\prime}(v)}{2 \Omega \vartheta_{1}^{2}(v)}\left[-J \frac{u}{2 \Omega^{2}} \vartheta_{1}^{\prime}(v)+\frac{\partial \vartheta_{1}(v)}{\partial \omega}\right]- \\
+J\left(\frac{1}{2 \Omega^{2}} \frac{\vartheta_{1}^{\prime}(v)}{\vartheta_{1}(v)}+\frac{u \eta}{\omega^{2}}\right)+\frac{u}{\Omega} \frac{\partial \eta}{\partial \omega} \\
\frac{\partial \wp(u)}{\partial \omega}=\frac{\partial e_{1}}{\partial \omega}+2\left(\wp(u)-e_{1}\right)\left[-\frac{J}{\Omega}+\frac{\partial \vartheta_{1}^{\prime}(0)}{\partial \omega} \frac{1}{\vartheta_{1}^{\prime}(0)}-\frac{\partial \vartheta_{2}(0)}{\partial \omega} \frac{1}{\vartheta_{2}(0)}\right] \\
+2\left(\wp(u)-e_{1}\right)\left[\begin{array}{c}
J \frac{u}{2 \Omega^{2}} \vartheta_{2}^{\prime}(v)+\frac{\partial \vartheta_{2}(v)}{\partial \omega} \\
-\frac{u}{\vartheta_{2}(v)} \vartheta_{1}^{\prime}(v)+\frac{\partial \vartheta_{1}(v)}{\partial \omega}
\end{array}\right]
\end{gathered}
$$

where $\Omega=\omega=\alpha-\beta$ and $J=1$ if $|\omega| \leq|\bar{\omega}|$, whereas $\Omega=\bar{\omega}=i \pi$ and $J=0$ if $|\omega|>|\bar{\omega}|$. 
2.3. Differentiation of $\mu(z)$. Let $\mu(z)$ be the chain of transformations which leads from the plane of the two adjacent circles $z$ to $\mu$, the plane of the concentric circles. Keeping $\alpha_{0}$ and $\varphi_{p}$ fixed, the mapping is uniquely defincd as a function of $x_{s}$ and $\varphi_{s}$. We derive the parameters (2.11), keeping a fixed velocity at infinity, with respect to $x_{s}$ and $\varphi_{s}$. These derivatives involve $a, b, r_{i}$ and $r_{e}$. It is $r_{i}=\mu(-1), r_{e}=\mu\left(x_{s}+r_{s}\right)$.

$$
\begin{aligned}
& \frac{\partial b}{\partial x_{s}}=b\left(\frac{2 x_{s}}{x_{s}^{2}-r_{s}^{2}+1}-\frac{1}{x_{s}}\right) \\
& \frac{\partial c}{\partial x_{s}}=\frac{\left(x_{s}-b\right)\left(1-\frac{\partial b}{\partial x_{s}}\right)}{c} \\
& \frac{\partial \alpha}{\partial x_{s}}=\frac{1-\frac{\partial b}{\partial x_{s}}+\frac{\partial c}{\partial x_{s}}}{x_{s}+r_{s}-b+c}-\frac{1-\frac{\partial b}{\partial x_{s}}-\frac{\partial c}{\partial x_{s}}}{x_{s}+r_{s}-b-c} \\
& \frac{\partial \beta}{\partial x_{s}}=\frac{\frac{\partial b}{\partial x_{s}}-\frac{\partial c}{\partial x_{s}}}{c-b-1}+\frac{\frac{\partial b}{\partial x_{s}}+\frac{\partial c}{\partial x_{s}}}{c+b+1} \\
& \frac{\partial \theta_{s}}{\partial x_{s}}=\operatorname{Im}\left(\frac{\partial \lambda_{s}}{\partial x_{s}}\right)=\operatorname{Im}\left(\frac{1-\frac{\partial b}{\partial x_{s}}+\frac{\partial c}{\partial x_{s}}}{z_{s}-b+c}-\frac{1-\frac{\partial b}{\partial x_{s}}-\frac{\partial c}{\partial x_{s}}}{z_{s}-b-c}\right) \\
& \frac{\partial \theta_{p}}{\partial x_{s}}=\operatorname{Im}\left(\frac{\partial \lambda_{p}}{\partial x_{s}}\right)=\operatorname{Im}\left(\frac{-\frac{\partial b}{\partial x_{s}}+\frac{\partial c}{\partial x_{i}}}{c-b+z_{p}}+\frac{\frac{\partial b}{\partial x_{s}}+\frac{\partial c}{\partial x_{s}}}{-c-b+z_{p}}\right) \\
& \frac{\partial \theta_{s}}{\partial \varphi_{s}}=\operatorname{Im}\left(\frac{\partial \lambda_{s}}{\partial \varphi_{s}}\right)= \\
& \operatorname{Im}\left(\frac{r_{s}\left(-\sin \varphi_{s}+i \cos \varphi_{s}\right)}{z_{s}-b+c}-\frac{r_{s}\left(-\sin 1 \varphi_{s}+i \cos \varphi_{s}\right)}{z_{s}-b-c}\right)
\end{aligned}
$$

whereas

$$
\frac{\partial \alpha}{\partial \theta_{s}}=\frac{\partial \beta}{\partial \theta_{s}}=\frac{\partial \theta_{p}}{\partial \theta_{s}}=0
$$

2.4. Circulation manifold. As we change $x_{s}$ and $\theta_{s}$ there are four remarkable situations. The lift may asymptoticaly increase or decrease with increasing distance between the two circles. In addition there may be a local maximum, or a local minimum. The variation of $\theta_{s}$ dces not give rise to any interesting change in the shape of the manifold, except in Fig. 2.10 where a local maximum of the circulation with respect to $\theta_{s}$ is shown. The different behaviors are due to the interplay between the radius of the secondary circle and $\theta_{p}$, as it is shown in the following figures. This is basically the reason for which this test case may be interesting: gradicnt based method would be not able to exit a local minima, whereas genetic algorithms can, see for example [7] . The case shown in Figs. 2.4-2.5 is for a secondary circle of radius $r_{s}=0.2, \theta_{p}=-10^{\circ}, \alpha_{0}=10^{\circ}$, $1.3 \leq x_{s} \leq 6.3,-10^{\circ} \leq \theta_{s} \leq 10^{\circ}$. Each of these intervals is divided in 20 segments and the circulation and 
its derivatives are evaluated at every resulting grid point. For this configuration lift is increasing with the distance, whereas in the case of Figs. 2.6-2.7 lift is decreasing with distance $\left(r_{s}=0.6, \theta_{p}=30^{\circ}, \alpha_{0}=10^{\circ}\right.$, $\left.1.6 \leq x_{s} \leq 6.6,-30^{\circ} \leq \theta_{s} \leq 30^{\circ}\right)$. In these figures and in the following the results are presented with respect to the grid points rather than explicitly with respect to the variable relative to the axis.

In Figs. 2.8-2.9 it is shown how with a proper choice of the parameters it is possible to obtain a local minimum $\left(r_{s}=0.05, \theta_{p}^{o}=-30, \alpha_{0}^{o}=10,1.15 \leq x_{s} \leq 6.15,-30^{\circ} \leq \theta_{s} \leq 30^{\circ}\right)$. In contrast, with $r_{s}=0.05$, $\theta_{p}^{o}=30, \alpha_{0}^{o}=10,1.15 \leq x_{s} \leq 6.15,-30^{\circ} \leq \theta_{s} \leq 30^{\circ}$; we have a local maximum, with respect to both $x_{s}$ and $\theta_{s}$ (see Figs. 2.10- 2.12).

We limited ourself to the explicit computation of the derivatives with respect to $x_{s}$ and $\theta_{s}$ in order to present conveniently the results and to keep a link with real world applications where these are basically the parameters to be varied. It is straight forward, using the derivatives of the elliptic functions, that we evaluate in the sections before, to determine the differential with respect to any other geometrical quantity on the plane of the adjacent circles.

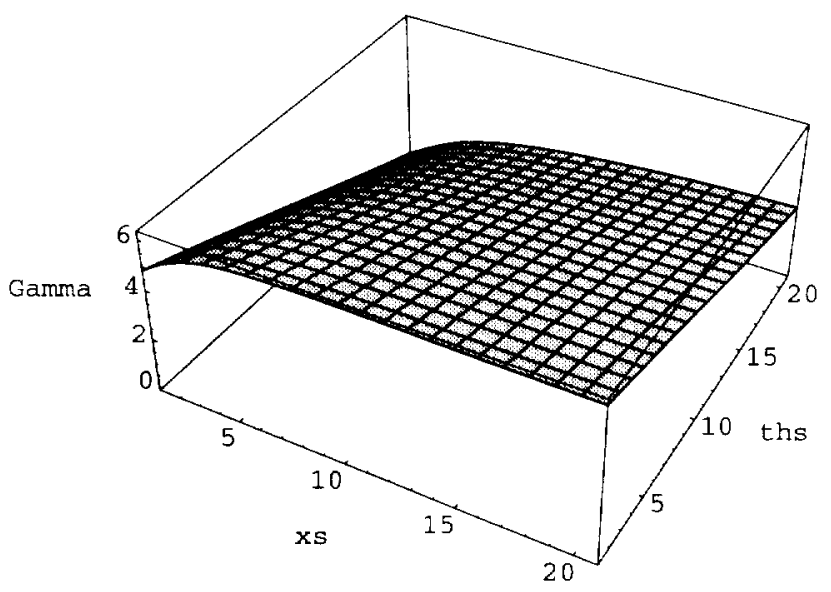

Fig. 2.4. Circulation as a function of $x_{s}$ (xs) and $\theta_{s}$ (ths): the circulation increases with $x_{s}$.

3. The Theodorsen-Garrik transform revisited. Here we adapt the Theodorsen-Garrik transform as proposed by Ives [6], in order to differentiate it by divided differences.

Our scope is to transform the domain exterior to two adjacent airfoils (see Fig. 3.1) on the plane $z_{f}$, into the region bounded by two concentric circles on the plane $z_{c}$. Let us first transform the primary profile into a nearly circular region by the Karman-Trefftz transform

$$
\left(\frac{z_{f}-z_{T p}}{z_{f}-z_{N p}}\right)^{\tau}=\frac{\zeta-1}{\zeta+1}
$$

with $\tau=\pi /\left(2 \pi-\delta_{p}\right), \delta_{p}$ being the trailing edge angle of the primary profile, $z_{T p}$ is its location, and $z_{N_{p}}$ is a point in the vicinity of the leading edge, inside the airfoil. By the Theodorsen transform the quasi circle on $\zeta$ is mapped onto a unit circle on $z$

$$
\zeta=z \exp \left\{\sum_{j=0}^{\infty}\left[\left(a_{j}+i b_{j}\right) z^{-j}\right]\right\}
$$




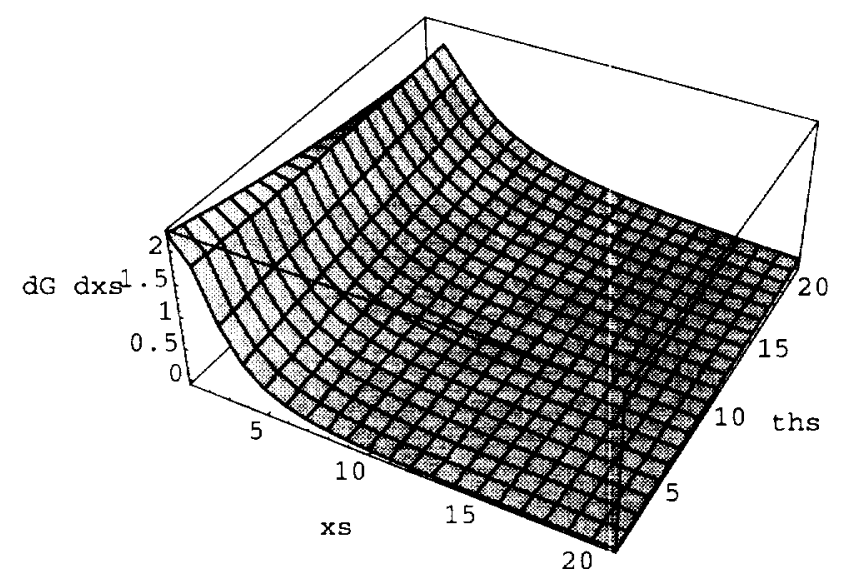

FIG. 2.5. Derivative of the circulation wiih respect to $x_{\mathrm{s}}$.

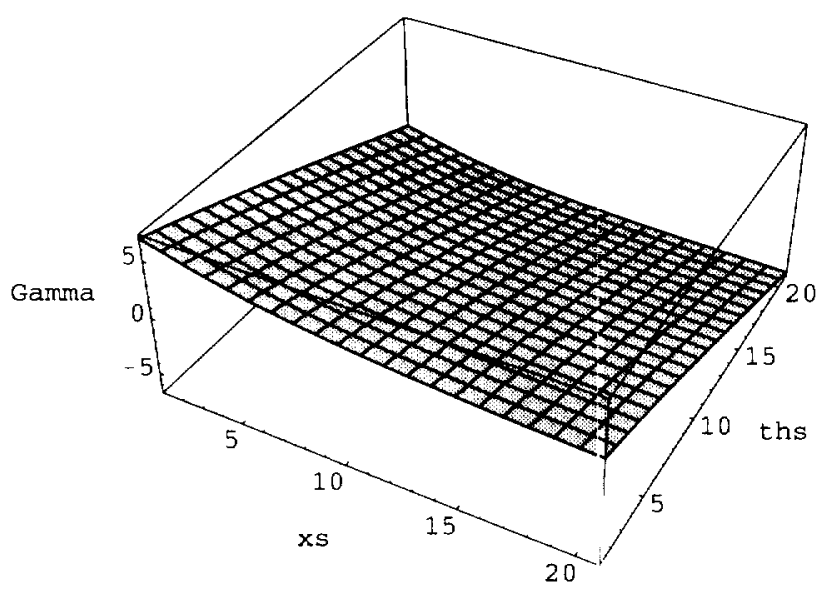

FIG. 2.6. Cinculation as a function of $x_{s}$ and $\theta_{s}$ : the (irculation decreases with $x_{s}$.

The coefficients $a_{j}$ and $b_{j}$ of the suitably truncated series in Eq. (3.2) are iteratively found by an FFT, imposing that the points of the unit circle on $z$ are mapped on the border of the nearly circular domain on $\zeta$. For the details see [6].

The sequence of mappings that transformed the primary profile into a unit circle acted on the secondary profile as well. Yet, the tangent discontinuity at the trailing edge of the secondary profile is still there, therefore it is necessary to map the plane $z$ so that the unit circle is unchanged and the image of the secondary profile is mapped onto a quasi circle. This is done by

$$
\frac{\xi-\xi_{T}}{\xi-\xi_{N}} \frac{\xi-1 / \xi_{T}^{*}}{\xi-1 / \xi_{N}^{*}}=\left(\frac{z-z_{T s}}{z-z_{N s}} \frac{z-1 / z_{T s}^{*}}{z-1 / z_{N s}^{*}}\right)^{\top}
$$

where $\tau=\pi /\left(2 \pi-\delta_{s}\right), \delta_{s}$ being the trailing edge angle of the secondary profile, $z_{T s}$ is the location on the plane $z$, and $z_{N s}$ is a point inside the secondary profile in the vicinity of the leading edge. The constants $\xi_{T}$ 


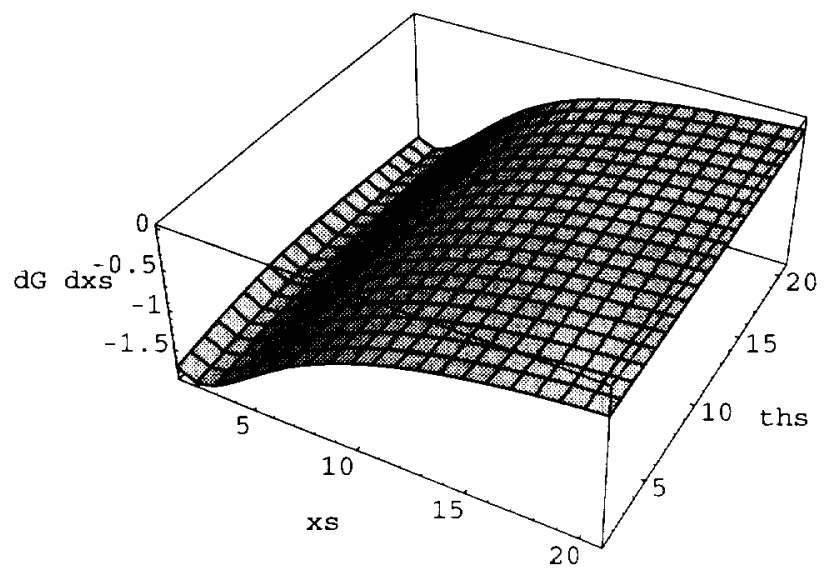

FIG. 2.7. Derivative of the circulation with respect to $x_{s}$

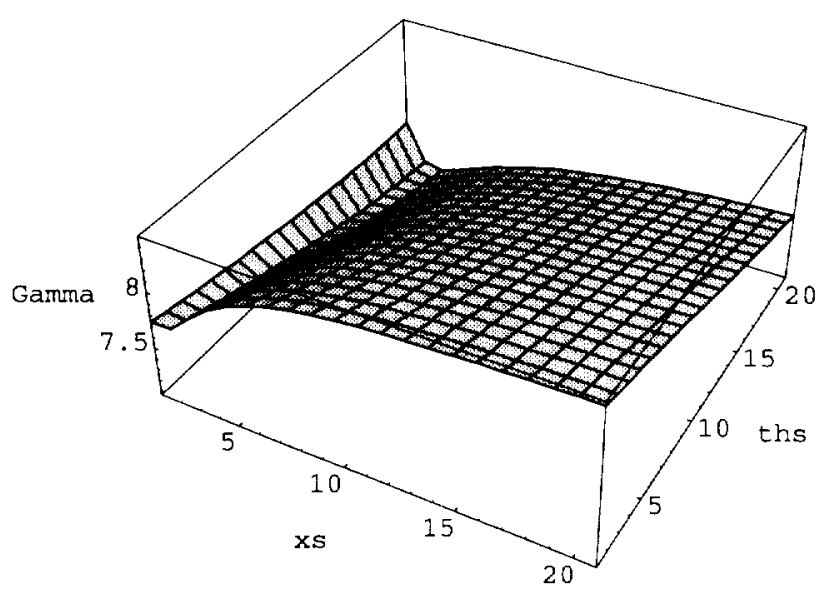

FIG. 2.8. Circulation as a function of $x_{s}$ and $\theta_{s}$ : the circulation has a local minimum.

and $\xi_{N}$ are found as follows. Let

$$
f(z)=\left(\frac{z-z_{T s}}{z-z_{N s}} \frac{z-1 / z_{T s}^{*}}{z-1 / z_{N s}^{*}}\right)^{\tau}
$$

and

$$
g(\xi)=\frac{\xi-\xi_{T}}{\xi-\xi_{N}} \frac{\xi-1 / \xi_{T}^{*}}{\xi-1 / \xi_{N}^{*}}
$$

The mappings $f(z)$ and $g(\xi)$ have the singularities $d f / d z=d g / d \xi=0$ for $f\left(z_{N s}\right)=g\left(\xi_{N}\right)$ and $f\left(z_{T s}\right)=$ $g\left(\xi_{T}\right)$. These equations were solved iteratively in $[6]$, whereas we found that they can be solved in a closed form.

At last, by the Theodorsen-Garrik transform it is possible to map $\xi$ on two concentric circles on the 


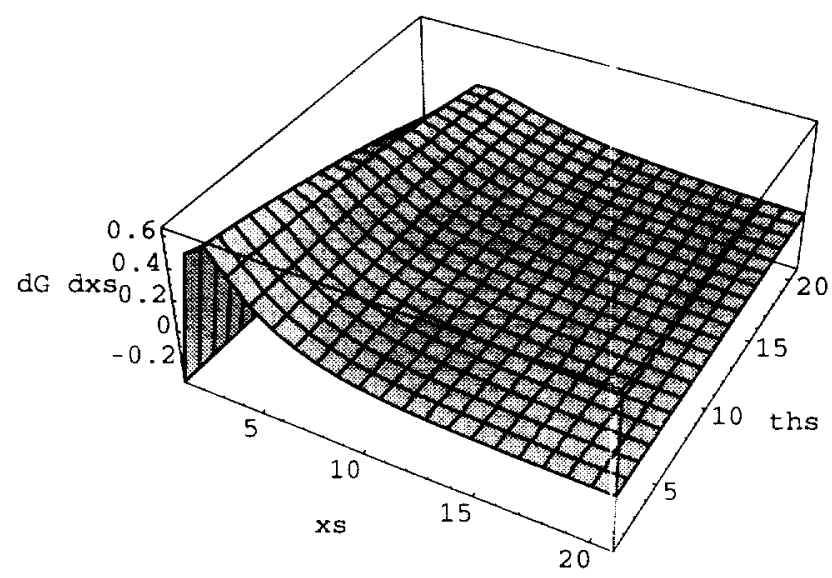

FIG. 2.9. Derivative of the circulation with respect to $x_{s}$.

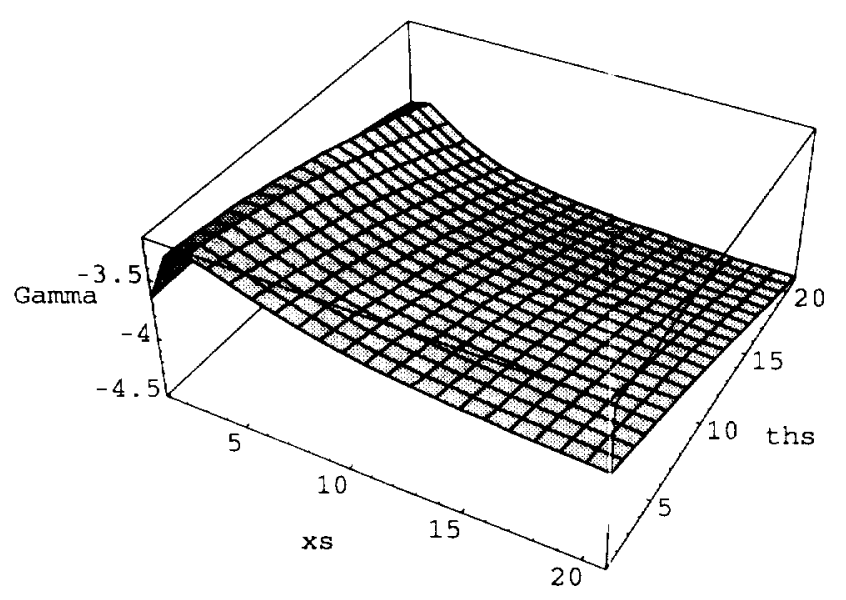

Fig. 2.10. Circulation as a function of $x_{s}$ and $\theta_{s}$ : the circulation has a local maximum.

plane $z_{c}$ :

$$
\xi=z_{c} \exp \left\{\sum_{j=0}^{\infty}\left[\left(-a_{j}^{\prime}+i b_{j}^{\prime}\right)\left(R z_{c}\right)^{-j}+\left(a_{j}^{\prime}+i b_{j}^{\prime}\right)\left(R / z_{c}\right)^{-j}\right]\right\}
$$

where $z_{c}=R \exp (i \theta)$. The radius $R$ and the coefficients $a_{j}^{\prime}$ and $b_{j}^{\prime}$ are obtained by a trial and error process based on an FFT, as for Eq. (3.2). For example in Fig.( 3.2-3.3) the pressure isolines and the streamlines, relative to the flow around two wing sections computed by this method, is presented.

3.1. Divided differences derivative. The set of transformations we described in the previous section is not easily differentiated explicitly, since the Theodorsen tranform and the Theodorsen-Garrick transform are defined implicitly, and the coefficients are determined by an iterative process. For this reason we decided to differentiate the mapping from the physical plane to the plane of the concentric circles by divided differences. Then, we used the results of the analytic differentiation of Section 2 in order to compute the gradient 




FIG. 2.11. Derivative of the circulation with respect to $x_{s}$

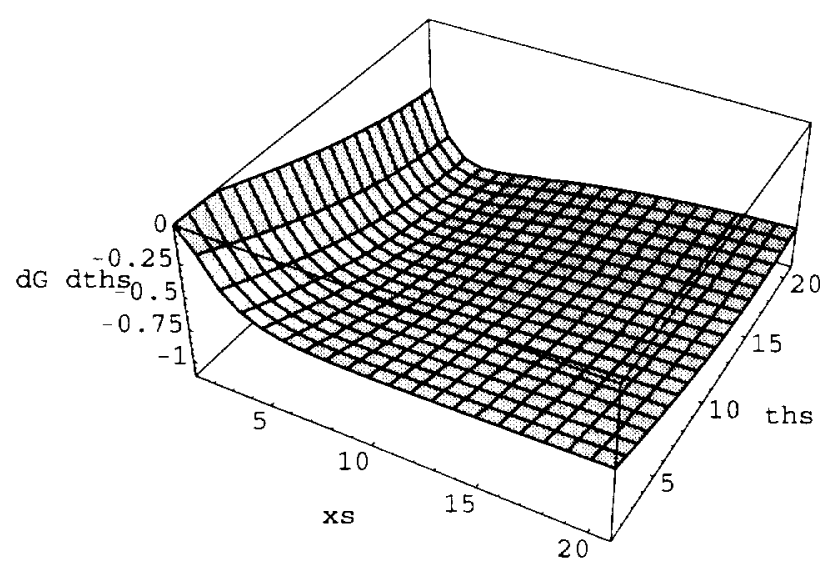

FIG. 2.12. Derivative of the circulation with respect to $\theta_{\mathbf{s}}$.

of the circulation with respect to the three parameters which define the position of the flap with respect to the main airfoil in the physical space, i.e., $\left(X_{s}, Y_{s}\right)$ the location of the leading edge of the flap and $\Theta$ its angle of attack.

As we mentioned in Section 2, all of the variables (2.11) defining the solution on the plane of the adjacent circles with focal points in \pm 1 are functions of $X_{s}, Y_{s}$ and $\Theta$. We computed the derivative of such functions by divided differences: for example we gave a small increment to $X_{s}$ and evaluate the increments of the variables (2.11) by means of the chain of mappings described in the section above. The differential is then obtained as the ratio between the increments of variables (2.11) and the increment in $X_{s}$.

As an example, we present the results obtained for the case of two adjacent NACA-0012 airfoils. The incidence of the flow at infinity is 10 degrees and the chord of the flap is 1/10 the chord of the main airfoil; also, the range for $X_{s}, Y_{s}$ and $1 \leq X_{s} \leq 1.3,-0.1 \leq Y_{s} \leq 0.1$ and $\Theta$ is and $-5 \leq \Theta \leq-2$. These intervals 


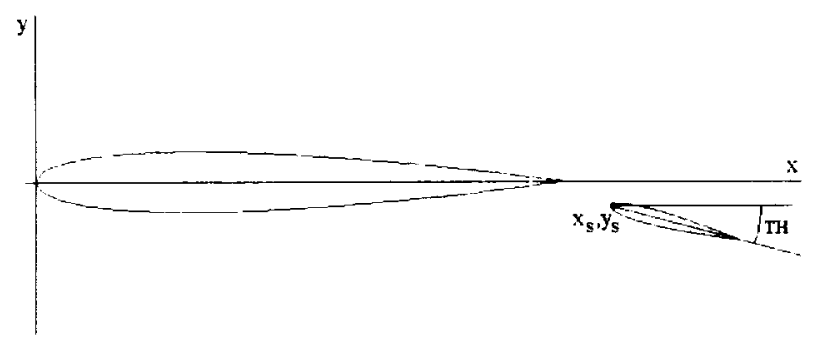

FIG. 3.1. Plane of the two airfoils.

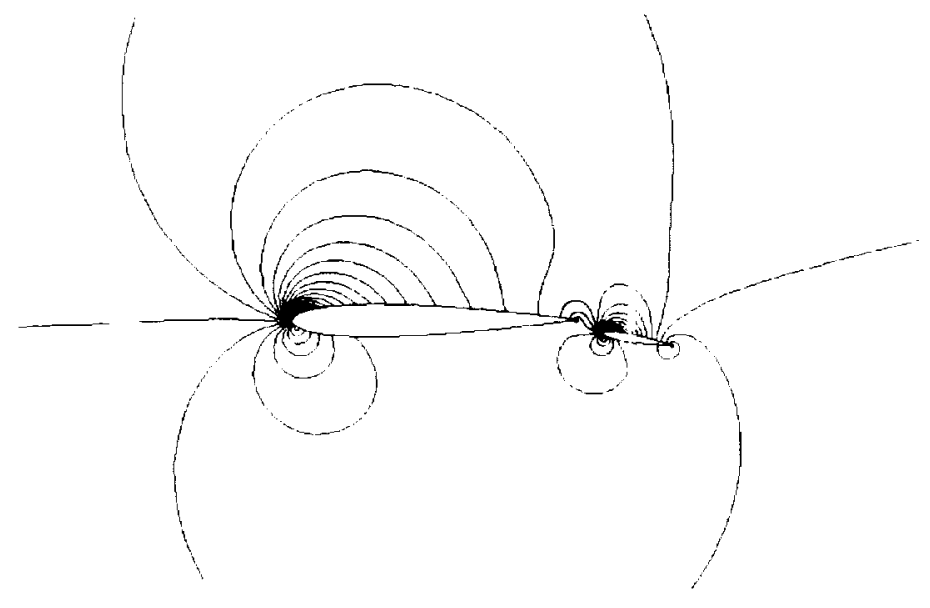

FIG. 3.2. Pressure field.

are subdivided on a mesh of 10 points. As it is seen in Fig. 3.4, there exist a non-monotone region of the circulation manifold. The circulation isosurfaces are plotted for increasing values of lift from top to bottom (0.71 to 0.74$)$. In Fig 3.5 the gradient is shown with respect to $\Theta$ computed as explained in the present section. The gradient values of the plotted isosurfaces range from 0 to $8 \cdot 10^{-3} \mathrm{from}$ left to right.

The features of the circulation manifold are influenced by the dimension of the flap as well as by the incidence of the flow at infinity. An abundance of different hehaviors may be obtained changing these parameters, as in the two adjacent circles case.

4. Conclusions. We presented two optimization test casıs. The first concerns the computation of the circulation functional for two adjacent circles, when the relative position and the trailing edge of the secondary circle are varied. The gradient with respect to those variables was computed analytically and results have been presented for several geometrical configurations. 

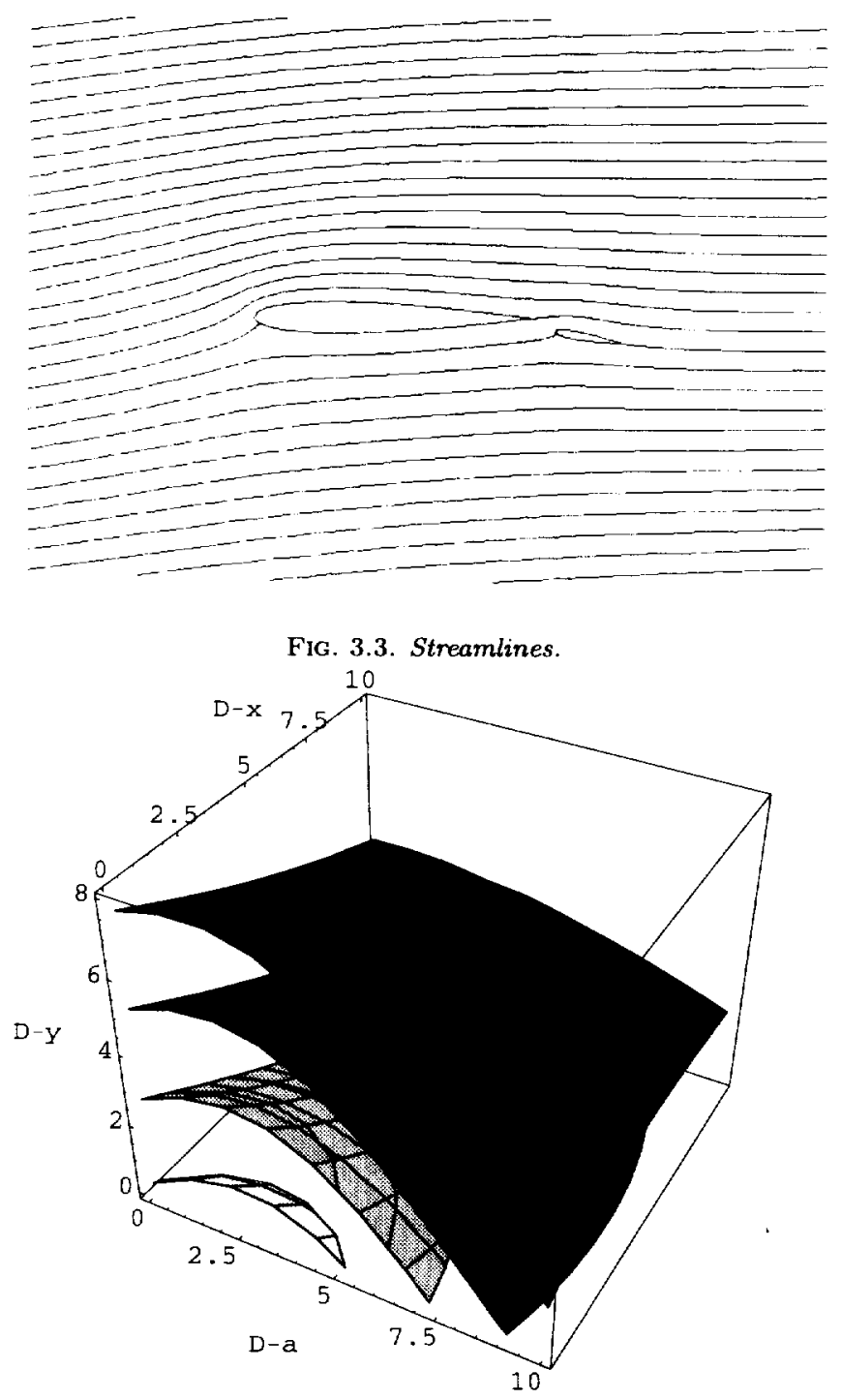

FIG. 3.4. Circulation isosurfaces: increasing values from top to bottom. $D-x$ is the increment in $X_{s}, D-y$ in $Y_{s}$ and $D$-a in $\Theta$

The second test case was related to the computation of the circulation functional for two adjacent airfoils, as the secondary airfoil is displaced. Since the mappings involved to compute the theoretical solution are defined implicitly, we decided to compute the gradient of such transformations by divided differences. Results showing the nature of the circulation manifold were presented.

In summary, it was shown that the space of solutions for such problems include non trivial situations of local minima and maxima. Such richness of behaviors and the relatively complicated geometrical optimization, which in the case of numerical solution involves remeshing and accurate definition of the gradient for moving boundaries, should be an interesting feature to test optimization algorithm of different nature.

The codes developed for this study are available at ICASE - NASA Langley Research Center, Hampton, VA. 


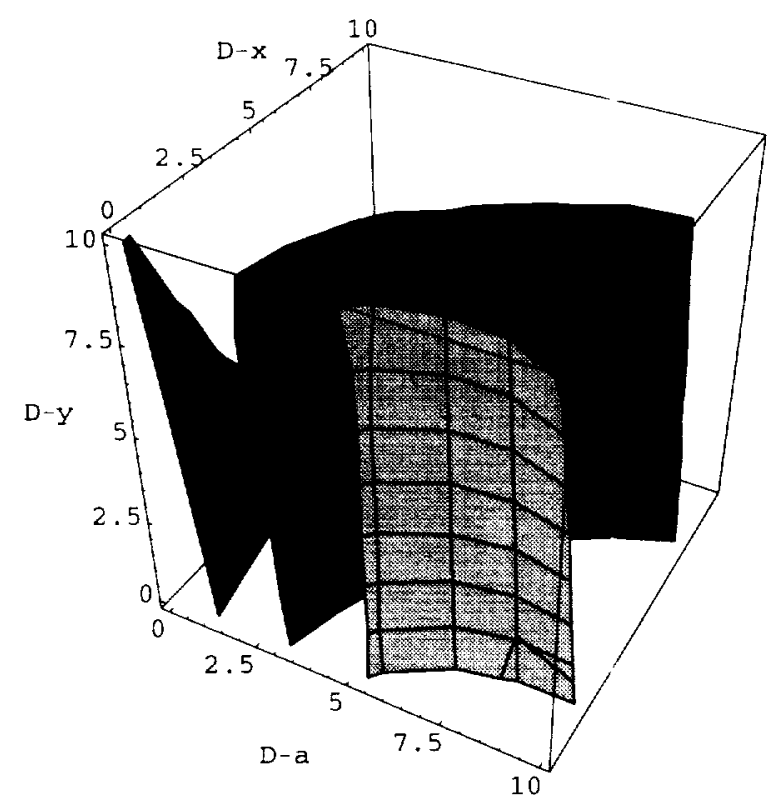

FIG. 3.5. Gradient isosurfaces. Gradient values from 0 to $8 \cdot 10^{-3}$ from left to right.

Aknowledgments. We would like to thank Manuel D. Salas, ICASE Director, for pointing out that the assumptions in [3] were erroneous, and hence, for motivating this work. Also, we are pleased to thank Professor Gino Moretti, N.Y. Polytechnic, for several valuable discussions resulting in original hints and remarks about the mapping of two profiles onto two circles and about an alternative derivation of the elliptic functions with respect to their period.

\section{REFERENCES}

[1] M. Lagally, Die reibungslose Strömung im Aussengebiet z:veier Kreise, ZAMM, Band 9, Heft 4, Aug. 1929, pp. 299-305.

[2] A. IOLlo AND M.D. SALAS, Optimum transonic airfoils based on the Euler equations, ICASE Report No. 96-76, 1996.

[3] B.R. Williams, An exact test case for the plane potential flcw about two adjacent lifting airfoils, R.A.E. Report No. 3717, 1971.

[4] C. Ferrari, Sulla trasformazione conforme di due cerchi in due profili alari, Memorie della Reale Accademia delle Scienze di Torino, Serie II, Vol LXVII, 1930, pp. 1-15.

[5] F. Tricomi, Funzioni ellittiche, Monografie di Matematica Applicata CNR, Zanichelli, 1951.

[6] D.C. Ives, A modern look at conformal mapping including multiply connected regions, AIAA Journal, 14, No. 8, 1976.

[7] N. Marco, C. Godart, J.A. Desideri, B. Mantel, and J. Périaux, A genetic algorithm compated with a gradient-based method for the solution of an active control model problem, INRIA Research report RR-2948, INRIA, France. 



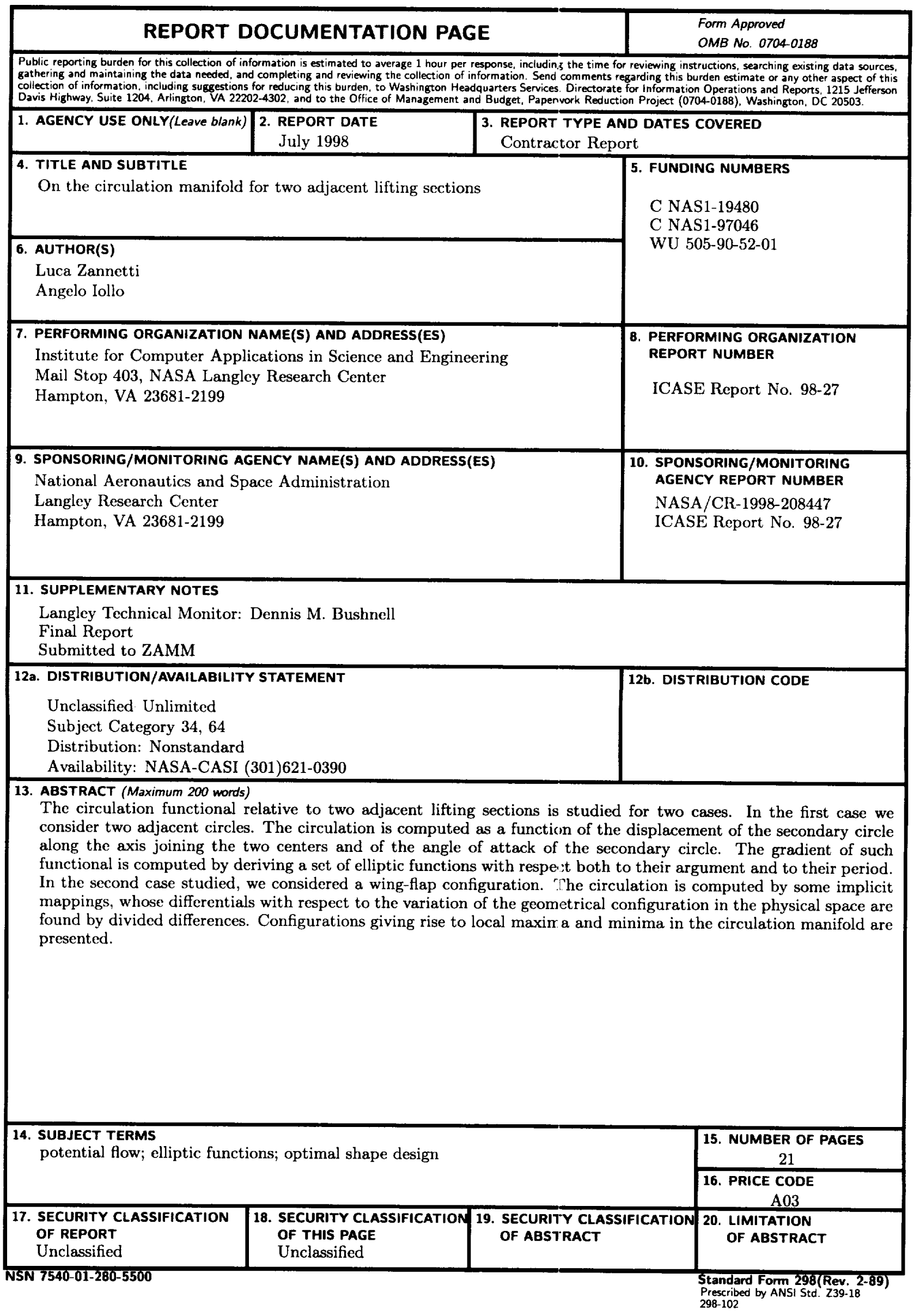

